\title{
Budget Impact of Enzalutamide for Nonmetastatic Castration-Resistant Prostate Cancer
}

\author{
Neil M. Schultz, PharmD, MS; Ken O'Day, PhD, MPA; \\ Rebecca Sugarman, BSc, MSc; and Krishnan Ramaswamy, PhD
}

\begin{abstract}
BACKGROUND: Prostate cancer is the most common cancer and secondleading cause of cancer death among men in the United States. Prostate cancer poses a large economic burden, which increases with progression from localized to metastatic disease. Newly approved treatments for nonmetastatic castration-resistant prostate cancer (nmCRPC) delay disease progression and reduce the risk of metastatic disease. Quantifying the potential budget impact of these new treatments is of interest to health care decision makers.
\end{abstract}

OBJECTIVE: To estimate the budget impact of enzalutamide for the treatment of patients with nmCRPC in the United States over a 3-year time horizon.

METHODS: An Excel-based model was developed to estimate the budget impact to a U.S. health plan of enzalutamide, a second-generation antiandrogen, as an add-on to androgen deprivation therapy (ADT) for the treatment of high-risk nmCRPC patients (prostate-specific antigen doubling time of $\leq 10$ months). Comparators include apalutamide $+A D T$, bicalutamide + ADT, and ADT only. The analysis includes treatment costs for nmCRPC and for treatment after progression to metastatic castration-resistant prostate cancer (mCRPC). The treated population size was estimated from epidemiological data and literature. Dosing, duration of therapy, and adverse event rates were based on package inserts and pivotal studies. RED B00K, Centers for Medicare \& Medicaid Services fee schedules, and literature were used to obtain costs of drugs, adverse events, and health care visits. Market shares were estimated for each comparator before and after enzalutamide adoption. A 1-way sensitivity analysis was performed to quantify the impact of parameter uncertainty.

RESULTS: In a hypothetical 1-million-member plan with $3 \%$ annual growth, it was estimated that there would be approximately 19 eligible incident nmCRPC patients in year 1, increasing to 20 eligible incident patients in year 3 . With an assumed market share of approximately $6 \%$ for enzalutamide in year 1, the budget impact would be $\$ 106,074$ ( $\$ 0.009$ per member per month [PMPM]). With a $26 \%$ enzalutamide share in year 3 , the budget impact would be $\$ 632,729$ ( $\$ 0.048$ PMPM). Cumulative budget impact to the health plan over 3 years is estimated to be $\$ 1,082,095$ ( $\$ 0.028$ PMPM). The increased cost of the treatment regimen is partly offset by reduced postprogression costs.

CONCLUSIONS: Treatment of nmCRPC patients with enzalutamide has a modest budget impact that is partly offset by delaying progression to mCRPC.

J Manag Care Spec Pharm. 2020;26(4):538-49

Copyright $\odot 2020$, Academy of Managed Care Pharmacy. All rights reserved.

\section{What is already known about this subject}

Prostate cancer is the most common malignancy and secondleading cause of cancer death in men in the United States.

Prostate cancer has a considerable economic burden in the United States, with $\$ 5.4$ billion in prostate cancer health care spending in 2013.

Enzalutamide, an androgen-receptor inhibitor, is indicated for the treatment of metastatic and nonmetastatic castration-resistant prostate cancer (nmCRPC).

\section{What this study adds}

This model estimates the budget impact of enzalutamide to inform the gap in contemporary information on the cost of nmCRPC and incorporates new treatments for nmCRPC that have strong clinical data and outcomes for patients.

In a hypothetical health plan with 1 million members, with 19-20 eligible incident patients with nmCRPC, incorporating enzalutamide had a modest budgetary impact of $\$ 0.028$ per member per month over 3 years.

$\mathrm{P}$ rostate cancer (PC) is the most common malignancy in men in the United States, affecting 1 in 9 men during their lifetimes. Estimates predict there will be 174,650 new PC cases and 31,620 deaths because of PC in 2019. ${ }^{1}$ PC is diagnosed at an average age of 66 years. ${ }^{2}$ Most men are initially diagnosed with localized or locally advanced hormonesensitive PC, which depends on testosterone for proliferation. However, nearly all patients progress to castration-resistant PC (CRPC) after the initiation of antihormonal therapy. ${ }^{3}$ It has been estimated that one third of all nonmetastatic CRPC (nmCRPC) patients will progress to metastatic CRPC (mCRPC) within 2 years of diagnosis. ${ }^{4}$ A recent meta-analysis including 12,712 male patients with PC provided evidence that in patients with localized PC, metastasis-free survival (MFS) is a strong surrogate for overall survival (OS); $42 \%$ of patients had an OS event and 45\% had an MFS event, resulting in a patient-level Kendall rank correlation of 0.91 and trial-level $\mathrm{R}^{2}$ of $0.86 .^{5}$ As MFS is predictive of OS in localized PC, recent therapeutic advances have centered on delaying the development of metastasis. ${ }^{5}$ 
The economic burden of PC is substantial. In 2013, personal health care spending on PC, as reported in the National Health Expenditure Accounts, totaled \$5.4 billion (2015 U.S. dollars), with the greatest annual PC-related costs incurred by the initial and terminal stages of disease. ${ }^{6,7}$ In advanced disease (CRPC), the development of metastasis is associated with a $>2$-fold increase in mean per-patient, annual all-cause health care costs. ${ }^{8}$ Thus, treatments that delay progression of nmCRPC to $\mathrm{mCRPC}$ may also reduce or delay the increase in costs associated with mCRPC.

The initial treatment of PC is guided by prognostic risk stratification and life expectancy. Options in the management of local or locally advanced disease include active surveillance, radical prostatectomy, and radiation therapy, while androgen deprivation therapy (ADT) is recommended for locally advanced or regional disease. Upon progression to CRPC, treatment is guided by prostate-specific antigen (PSA) doubling time and the risk of metastasis. In nmCRPC, secondary antihormonal therapy has served as the treatment mainstay to prevent the onset of metastasis; however, second-generation antiandrogens as an adjunct to ADT have recently been approved for use in nmCRPC on the basis of improved MFS and are included in treatment guidelines. ${ }^{9}$

Enzalutamide (Xtandi, Astellas Pharma and Pfizer) is an androgen-receptor inhibitor, first approved for treatment of mCRPC in 2012, followed by an expanded indication to include nmCRPC, based on subsequent clinical trial data. ${ }^{10}$ Enzalutamide has been shown to extend median OS in patients with mCRPC before chemotherapy (hazard ratio [HR] for death $=0.71 ; P<0.001$ [PREVAIL] $)$ and following chemotherapy (HR for death $=0.63 ; P<0.001$ [AFFIRM]). ${ }^{11,12}$ In the PROSPER phase 3 randomized controlled trial comparing enzalutamide to placebo as an adjunct to ADT in patients with high-risk nmCRPC, defined as a PSA doubling time of $\leq 10$ months, enzalutamide extended median MFS ( $\mathrm{HR}=0.29 ; P<0.001)$, time to subsequent antineoplastic therapy $(\mathrm{HR}=0.21 ; P<0.001)$, and time to PSA progression $(\mathrm{HR}=0.07 ; P<0.001){ }^{13}$

As new therapies for PC enter the market, health care decision makers face a significant challenge in allocating scarce financial resources in an effort to improve population health. Budget impact models employ clinical and economic data to serve as 1 resource in the evaluation of new health technologies. With this purpose in mind, we developed a model to estimate the budget impact of enzalutamide used in combination with ADT for the treatment of patients with high-risk nmCRPC.

\section{Methods}

\section{Overview}

A model based in Excel (Microsoft, Redmond, WA) was developed from a U.S. third-party payer perspective for a hypothetical health plan with a population of 1 million members. The model estimates the budget impact of enzalutamide plus ADT for the treatment of patients with high-risk nmCRPC over a 3-year time horizon. Comparators include apalutamide plus ADT, bicalutamide plus ADT, and ADT only. Costs in the model took into account drug regimen, monitoring and adverse events, medical professional visits, and disease progression for patients who develop mCRPC. Patients with nmCRPC were assumed to continue on the initiated treatment until progression to mCRPC, at which point they switched to a treatment for mCRPC. Patients were assumed to remain on this treatment until death. Parametric survival curves from the PROSPER and SPARTAN phase 3 trials for enzalutamide and apalutamide, respectively, in nmCRPC patients were created using MFS, and the area under the curve was used to calculate the average number of months of treatment per patient per year (PPPY) under the assumption that patients would be treated until they develop metastatic disease. ${ }^{13-15}$ Figure 1 provides a schematic of the budget impact model structure. Key model assumptions are provided in Appendix A (available in online article).

The model was developed in accordance with guidelines and recommendations from the Academy of Managed Care Pharmacy and the International Society for Pharmacoeconomics and Outcomes Research. ${ }^{16-18}$ Per these guidelines, no discounting was used in the budget impact analysis.

This study used publicly available secondary data with no individual patient identifiers so is exempt from institutional review board review under 45 CFR 46.101(b).

\section{Model Inputs}

Target Population. The target population includes newly incident patients with high-risk nmCRPC based on the patient population studied in the PROSPER trial. To estimate the size of the treated patient population, the model applies the following series of filters to the size of the health plan population: proportion male, incidence of PC, proportion of PC cases that develop CRPC, proportion of CRPC cases that develop nonmetastatic disease, and the proportion of nmCRPC that are considered high risk (defined as PSA doubling time of $\leq 10$ months). The inputs used to estimate the size of the target population of high-risk nmCRPC patients are shown in Table 1. The model assumes a 3\% annual rate of population growth and a hypothetical 1-million-member plan. To accurately estimate the population size, the target population was stratified by age ( $<65$ years and $\geq 65$ years) to reflect the higher incidence of PC in older men.

Treatment Costs and Duration. Drug dosing was obtained from the prescribing information for each product. Medication unit prices for comparator drugs and concomitant medications were obtained from the wholesale acquisition costs from RED BOOK. ${ }^{19}$ Drug costs used in this model were current at the time that this analysis was completed (July 2018). 


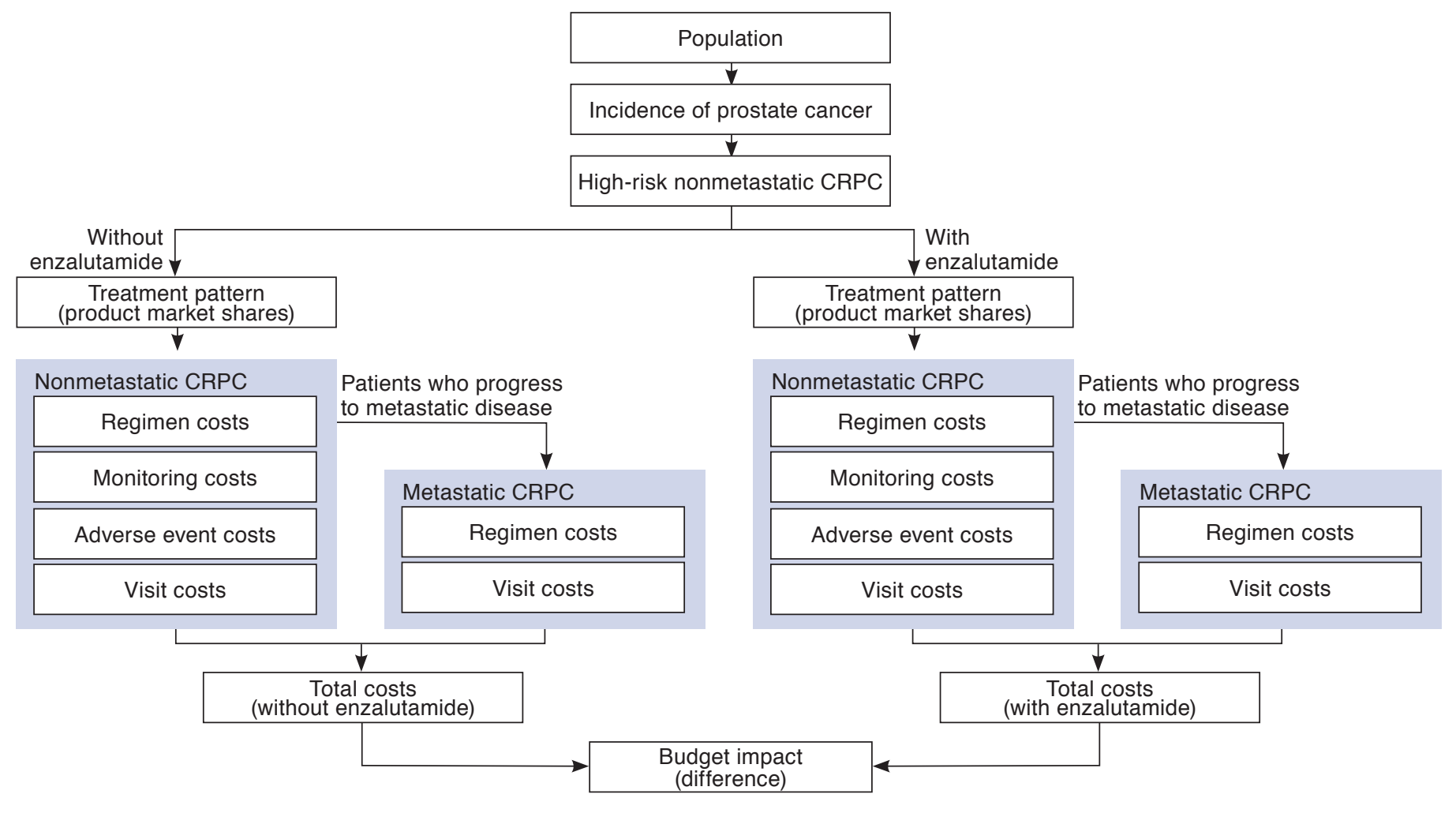

$C R P C=$ castration-resistant prostate cancer .

Administration costs for injections and monitoring laboratory costs were obtained from the Centers for Medicare \& Medicaid Services fee schedules. Oral medications were assumed to not incur any administration costs.

Costs of treatment regimens for nmCRPC and mCRPC were estimated separately. Patients with nmCRPC received either ADT alone or ADT in addition to enzalutamide, apalutamide, or bicalutamide. Included treatments were based on clinical practice patterns and product indications; treatments with low use in the United States were not included. Treatment costs for patients with nmCRPC were assumed to apply during the period of MFS based on clinical trial data. Median MFS, obtained from clinical trials, was 36.6 months and 40.5 months for enzalutamide and apalutamide, respectively. ${ }^{13,14}$ Median MFS for treatment with ADT alone was obtained from the control arm of the PROSPER trial (14.7 months) and median MFS for bicalutamide was assumed to be identical to that for ADT, given a similar HR for both treatments compared with enzalutamide in the STRIVE and PROSPER trials (HR=0.24 for bicalutamide and HR=0.29 for ADT, respectively). ${ }^{13}$ Using the MFS values, parametric survival curves were created assuming a constant failure rate (i.e., exponential function) in the model. The area under the curve was used to calculate the average number of months of treatment PPPY over the 3-year modeled time horizon. All patients were assumed to receive the same ADT treatment: leuprolide acetate.

When patients develop metastatic disease, a weighted average treatment cost per month was estimated based on market share assumptions for treatments indicated for metastatic disease (treatment [weight]: enzalutamide [30\%], abiraterone acetate [40\%], cabazitaxel [10\%], docetaxel [15\%], and radium-223 dichloride [5\%]). Weights were adjusted to exclude enzalutamide for patients receiving enzalutamide initially for the treatment of nmCRPC, since the drug is approved for both mCRPC and nmCRPC.

During the period when patients are receiving treatment for metastatic disease, an annual mortality rate of $21.7 \%$ was applied (no survival differences are assumed among metastatic PC treatments) based on OS of patients with metastatic disease. ${ }^{20}$

Monitoring and Adverse Event Costs. Monitoring tests and the annual frequency of each were based on clinical experts and prescribing information for each treatment. Monitoring costs for hepatic function panel, metabolic panel, PSA testing, 


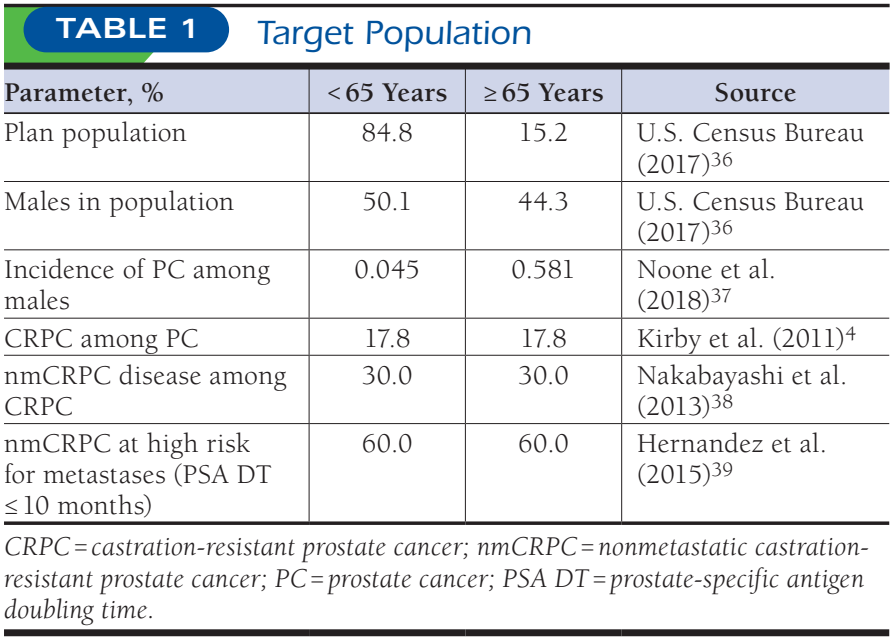

and monitoring and management for fracture risk with bone scan and use of bone-targeted agents were based on the Centers for Medicare \& Medicaid Services clinical laboratory and physician schedules, and RED BOOK. ${ }^{19,21,22}$ Cost per resource was multiplied by its annual use to calculate the total cost of monitoring over the duration of treatment for each model comparator. Frequency of monitoring tests and unit costs are included in Table 2.

Adverse event rates were obtained from product prescribing information and key pivotal trials. The model included grade 3 and grade 4 adverse events with $a \geq 1 \%$ incidence rate, and assumed that patients experiencing these events would require hospitalization, in accordance with the National Cancer Institute Common Terminology Criteria for Adverse Events. ${ }^{23}$ The costs associated with these events were obtained from the Healthcare Cost and Utilization Project Nationwide Inpatient Sample, except for the cost of a fall, which used numbers from the literature. ${ }^{24,25}$ Appendix B (available in online article) provides a detailed list of included adverse events, frequencies, and associated costs. As a weighted average cost of all mCRPC treatments was used to simplify the model, monitoring costs and adverse event costs typically associated with specific treatments were not included in the total mCRPC cost estimates.

Medical Professional Visit Costs. These costs included office visits, emergency department visits, and inpatient visits with an annual frequency from literature of $12.1,0.5$, and 0.2 visits per year, respectively, for nmCRPC and 21.9, 1.5, and 1.2 visits per year, respectively, for mCRPC. ${ }^{8}$ Unit costs for medical professional visits are $\$ 74.16$ for office visits, $\$ 1,712$ for emergency department visits, and $\$ 14,616$ for inpatient visits. ${ }^{22,24}$ Where necessary, costs were inflated to 2018 U.S. dollars using the medical care component of the Bureau of Labor Statistics Consumer Price Index. ${ }^{26}$
Market Share. Current market share projections without enzalutamide were based on the assumption that recently approved apalutamide+ADT use would increase over time, resulting in a reduction in usage of other treatments. For market share projections following U.S. Food and Drug Administration approval, the model assumed $\sim 6 \%$ market adoption of enzalutamide in year 1 , increasing to a projected $\sim 26 \%$ use in year 3. New market shares for competitor products were based on the assumption that market adoption of enzalutamide corresponded with a reduction in market shares of other products in proportion to their current market share. Market share estimates (Appendix C, available in online article) were based on manufacturer internal market research used for the purposes of this modeling only and may not necessarily be reflective of actual market share since third-party data are not yet publicly available.

\section{Model Outputs}

Budget Impact. The total cost of treatment for each therapy was the sum of the drug regimen costs, monitoring and adverse event costs, medical professional visit costs, and costs associated with disease progression for patients who develop mCRPC. The budget impact analysis calculated the difference in costs between the market with enzalutamide as treatment for nmCRPC and the current market in terms of the aggregate and per member per month (PMPM) expenditures.

Sensitivity Analyses. To test the model's sensitivity to variation in the model parameters, univariate sensitivity analyses were conducted for all key model parameters, with individual parameters being varied over a range from low to high, based on increasing and decreasing the values by $20 \%$. The output for the 1-way sensitivity analysis was the aggregate budget impact (difference in total costs with enzalutamide vs. without enzalutamide). Based on the clinical evidence that apalutamide and enzalutamide have demonstrated similar efficacy in clinical trials and an indirect comparison found no evidence of a significant difference in this outcome, a scenario analysis was also conducted in which the median MFS for enzalutamide and apalutamide were both set as equal. ${ }^{27}$

\section{Results}

\section{Budget Impact}

In a hypothetical 1-million-member plan with 3\% annual growth, it was estimated that there would be approximately 19 eligible incident nmCRPC patients in year 1, increasing to 20 in year 3. Based on the scenario detailed, the budget impact associated with the introduction of enzalutamide for the treatment of nmCRPC in year 1 would be $\$ 106,074$ ( $\$ 0.009$ PMPM); in year 3 , with an increased enzalutamide share, the budget impact would be $\$ 632,729$ ( $\$ 0.048$ PMPM). Cumulative budget impact to the health plan over 3 years is estimated to 


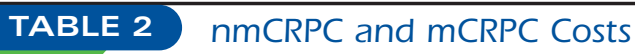

\begin{tabular}{|c|c|c|c|c|}
\hline & Enzalutamide & Apalutamide & Bicalutamide & ADT \\
\hline Dose & $160 \mathrm{mg} / \mathrm{day}$ & $240 \mathrm{mg} / \mathrm{day}$ & $50 \mathrm{mg} / \mathrm{day}$ & $22.5 \mathrm{mg} / 3$ months \\
\hline Cost per package, ${ }^{\mathrm{b}} \$$ & 10,905 & 10,920 & 15 & 1,355 \\
\hline Doses per package & 30 & 30 & 30 & 1 \\
\hline Administration frequency & Daily oral & Daily oral & Daily oral & 3-month injectiona \\
\hline Cost per month, \$ & 11,057 & 11,072 & 15 & $459 \mathrm{c}$ \\
\hline Adverse event cost per month, ${ }^{\mathrm{d}} \$$ & 54 & 52 & 89 & 61 \\
\hline Monitoring cost per month, $\$$ & 59 & 59 & 13 & 0 \\
\hline nmCRPC HRU cost per month, \$ & 390 & 390 & 390 & 390 \\
\hline Weighted mCRPC regimen cost, ${ }^{\mathrm{e}} \$$ & 9,535 & 9,740 & 9,740 & 9,740 \\
\hline mCRPC HRU cost per month, $\$$ & 1,811 & 1,811 & 1,811 & 1,811 \\
\hline
\end{tabular}

anformation on drug dosing was obtained from the prescribing information for each respective product. ${ }^{10,41-43}$

${ }^{b}$ Cost was based on wholesale acquisition cost because average selling price was not available.

${ }^{c}$ Cost per month includes an administration cost of $\$ 20.88$ based on the Centers for Medicare E Medicaid Services 2018 physician fee schedule (Current Procedural

Terminology code 96372 subcutaneous or intramuscular injection). ${ }^{22}$

${ }^{\mathrm{C}}$ Cost per month was based on duration of treatment.

${ }^{e}$ For calculation of daily drug costs for docetaxel, where dosing is based on weight or BSA, patients were assumed to have an average weight of $88.4 \mathrm{~kg}$ and a BSA of $2.1 \mathrm{~m}{ }^{2}$ based on literature values. ${ }^{40}$ Costs for metastatic patients receiving cabazitaxel and radium-223 dichloride are based on a full single-use vial, so any wastage is accounted for in the costs of these drugs. For metastatic patients receiving docetaxel, no wastage was included given the lower cost of this drug.

$A D T=$ androgen deprivation therapy; $B S A=$ body surface area; $H R U=$ health care resource utilization; $m C R P C=$ metastatic castration-resistant prostate cancer; nmCRPC = nonmetastatic castration-resistant prostate cancer.

be $\$ 1,082,095$ (\$0.028 PMPM). The increased cost of the treatment regimen is partially offset by reduced postprogression costs. The potential budget impact over this 3-year time horizon is shown in Table 3. See the appendices for supplemental budget impact results.

\section{Sensitivity Analysis}

A 1-way sensitivity analysis indicated that the model was most sensitive to the drug cost per month of the enzalutamide regimen, total population size, and enzalutamide use. The maximum potential budget impact, observed when the cost of enzalutamide was increased by $20 \%$, was $\$ 1,401,395$ and \$0.037 PMPM (Figure 2).

\section{Scenario Analysis}

In the scenario analysis in which the median MFS for enzalutamide and apalutamide were both set to 36.6 months (from enzalutamide's PROSPER trial), the result was a negligible change in the budget impact: 3-year cumulative budget impact was $\$ 1,083,309$ ( $\$ 0.028$ PMPM), a difference of $0.1 \%$ from the base case results.

\section{Discussion}

Until recently, patients with nmCRPC at risk of progression to mCRPC had limited treatment options. ${ }^{14,28}$ Recent trials for enzalutamide and apalutamide demonstrated the safety and effectiveness of these 2 antiandrogens in improving MFS., $3,13,14$ Individual trial data also suggest an improvement in OS, but these data are not fully mature; however, a recent meta-analysis including data from both trials found a significant increase in OS (HR $=0.76 ; 95 \%$ confidence interval $=0.59-0.76) .{ }^{29}$

As add-on therapies to ADT, the availability of enzalutamide and apalutamide will increase treatment costs, but the resulting delays in progression to mCRPC include some associated offsets in cost. Given the strong clinical outcomes and evidence supporting treatment in this patient population, it will be important for payers to understand the potential health economic implications of their utilization. To date, there have been few health economic studies of nmCRPC, as enzalutamide and apalutamide were only recently approved by the U.S. Food and Drug Administration in 2018. A recently published systematic review of cost and cost-effectiveness analyses in PC identified 38 studies, none of which examined the cost-effectiveness or budget impact of enzalutamide or apalutamide for nmCRPC..$^{30}$

However, there has been a substantial number of health economic studies in mCRPC. This is important to demonstrate how these new medications can reduce the risk of progression and thus prevent costs associated with poor disease outcomes. For example, a model on number needed to treat compared enzalutamide versus abiraterone plus prednisone in patients with $\mathrm{mCRPC}$ and found that enzalutamide resulted in lower treatment costs and better outcomes, despite having a higher acquisition cost. ${ }^{31}$ In PC, costs for terminal care per year are greater than costs for initial and continuing care; thus, by delaying progression, these medications can reduce health system costs in other areas. ${ }^{7}$

This model was developed to estimate the budget impact of enzalutamide for the treatment of nmCRPC from a U.S. third-party payer perspective. The model included drug 


\begin{tabular}{l|c|c|c}
\hline TABLE 3 & $\begin{array}{c}\text { Budget and Budget Impact over 3-Year } \\
\text { Time Horizon for Treatment of nmCRPC }\end{array}$ \\
\hline & $\begin{array}{c}\text { Current Market } \\
\text { Without } \\
\text { Enzalutamide, } \$\end{array}$ & $\begin{array}{c}\text { New Market } \\
\text { with } \\
\text { Enzalutamide, } \$\end{array}$ & $\begin{array}{c}\text { Budget } \\
\text { Impact, } \$\end{array}$ \\
\hline nmCRPC regimen & $2,043,882$ & $3,356,754$ & $1,312,872$ \\
\hline nmCRPC monitoring & 11,481 & 18,029 & 6,548 \\
\hline nmCRPC adverse & 54,877 & 55,011 & 134 \\
events & 320,894 & 332,068 & 11,175 \\
\hline nmCRPC visits & $3,930,592$ & $3,720,159$ & $-210,433$ \\
\hline mCRPC regimen & 730,796 & 692,595 & $-38,201$ \\
\hline mCRPC visits & $7,092,522$ & $8,174,616$ & $1,082,095$ \\
\hline Aggregate total & - & - & 0.028 \\
\hline PMPM & \multicolumn{4}{|c|}{-} & \\
\hline $\begin{array}{l}\text { mCRPC = metastatic castration-resistant prostate cancer; } \text { nmCRPC }=\text { nonmetastatic } \\
\text { castration-resistant prostate cancer; PMPM = per member per month. }\end{array}$
\end{tabular}

costs, administration costs, monitoring and management costs, adverse event costs, and medical professional visit costs, and accounted for the costs of disease progression from nmCRPC to $\mathrm{mCRPC}$ and death, based on patient survival curves. In a hypothetical health plan of 1 million members, the estimated cumulative 3-year total budget impact was $\$ 1,082,095$ : $\$ 9,198$ PPPY and $\$ 0.028$ PMPM. The total annual budget impact was $\$ 106,074$ (\$0.009 PMPM) in year 1 , increasing to $\$ 632,729$ (\$0.048 PMPM) in year 3. Treatment regimen costs for nmCRPC were the largest contributor to the increased budget, reflecting the difference in drug cost between enzalutamide plus ADT as compared with bicalutamide plus ADT or ADT alone. However, the budget increase was partially offset by reductions in mCRPC regimen costs and $\mathrm{mCRPC}$ medical professional visits because of delayed progression to metastatic disease.

A previous budget impact analysis of enzalutamide for the treatment of chemotherapy-naive patients with mCRPC estimated a 1-year budget impact of $\$ 0.04$ PMPM. $^{32}$ The current model estimates a notably lower 1-year budget impact for enzalutamide in nmCRPC of $\$ 0.009$ PMPM. The difference in these estimates is primarily because of differences in the patient population estimates and the projected use of enzalutamide.

The Institute for Clinical and Economic Review (ICER) recently conducted an assessment of apalutamide and enzalutamide for nmCRPC, including both a cost-effectiveness analysis and budget impact analysis. ${ }^{33}$ In its review of the clinical evidence, ICER concluded that both enzalutamide and apalutamide substantially delay disease progression and improve OS. For the cost-effectiveness analysis, ICER's assessment estimated incremental cost-effectiveness ratios of $<\$ 100,000$ per quality-adjusted life-year for both treatments compared with ADT alone, representing reasonable value for money. ${ }^{33}$ However, in addition to understanding the economic value of these treatments, payers may be interested in the potential budget impact because of their increased use among nmCRPC patients and the cost offsets because of delaying disease progression. In this model, delaying progression had a substantial positive effect on costs by lowering monthly health care resource utilization for patients with nmCRPC compared with mCRPC, in agreement with published literature. ${ }^{8}$

The ICER budget impact analysis estimated the annual incremental cost per patient of enzalutamide plus ADT versus ADT alone over a 5 -year time horizon as $\$ 64,751$. Following the ICER methodology, this model was reconfigured to conduct this same analysis and estimated an annual incremental cost per patient of $\$ 72,536$ for enzalutamide plus ADT versus ADT alone. The slightly higher cost estimates in our analysis result from using the primary endpoint of MFS to model progression from nmCRPC to mCRPC, which results in a longer duration of therapy for patients receiving nmCRPC treatment. The ICER budget impact analysis estimated that $11 \%$ of patients with nmCRPC could be treated with enzalutamide while remaining under ICER's stipulated \$991 million annual budget impact threshold. ICER also estimated that up to $18 \%$ of nmCRPC patients could be treated with enzalutamide under the annual budget impact threshold at a discounted wholesale acquisition cost, amounting to a $29 \%$ reduction in treatment cost.

However, these estimates depend on the size of the modeled patient population. ICER obtained its target population estimate from a recently published PC dynamic progression model. ${ }^{34}$ According to this analysis, the estimated annual incidence in the United States of nmCRPC is 59,000 patients. Our model applies figures from the literature to estimate the annual incidence of nmCRPC patients with PSA doubling time of $<10$ months (for consistency with the studied clinical trial populations), resulting in a substantially more conservative estimate of 6,500 patients annually in the United States. Removing the population filter for PSA doubling time of $<10$ months results in an annual incidence estimate of approximately 11,000 nmCRPC patients in the United States in our model.

There are limited epidemiologic studies available to estimate the size of the nmCRPC population. A previous analysis estimated a 5-year prevalence of 39,000 patients with nmCRPC in the United States. ${ }^{35}$ Based on this estimate, and using a median survival of 3 years, the incidence would be approximately 13,000 nmCRPC patients annually. Using our estimated nmCRPC population, it would be possible to treat $>40 \%$ of patients with enzalutamide and remain under ICER's $\$ 991$ million annual budget impact threshold. Aside from the cost of enzalutamide, the 1-way sensitivity analysis was most sensitive to parameters used to estimate the size of the target population and the market shares of enzalutamide. Additional consideration regarding the size of the eligible nmCRPC treatment population is warranted. For instance, payers may want to run analyses from their actual patient populations to identify the specific number of treatment-eligible patients in their health plans to inform budget impact analyses. 
FIGURE 2 Sensitivity Analysis

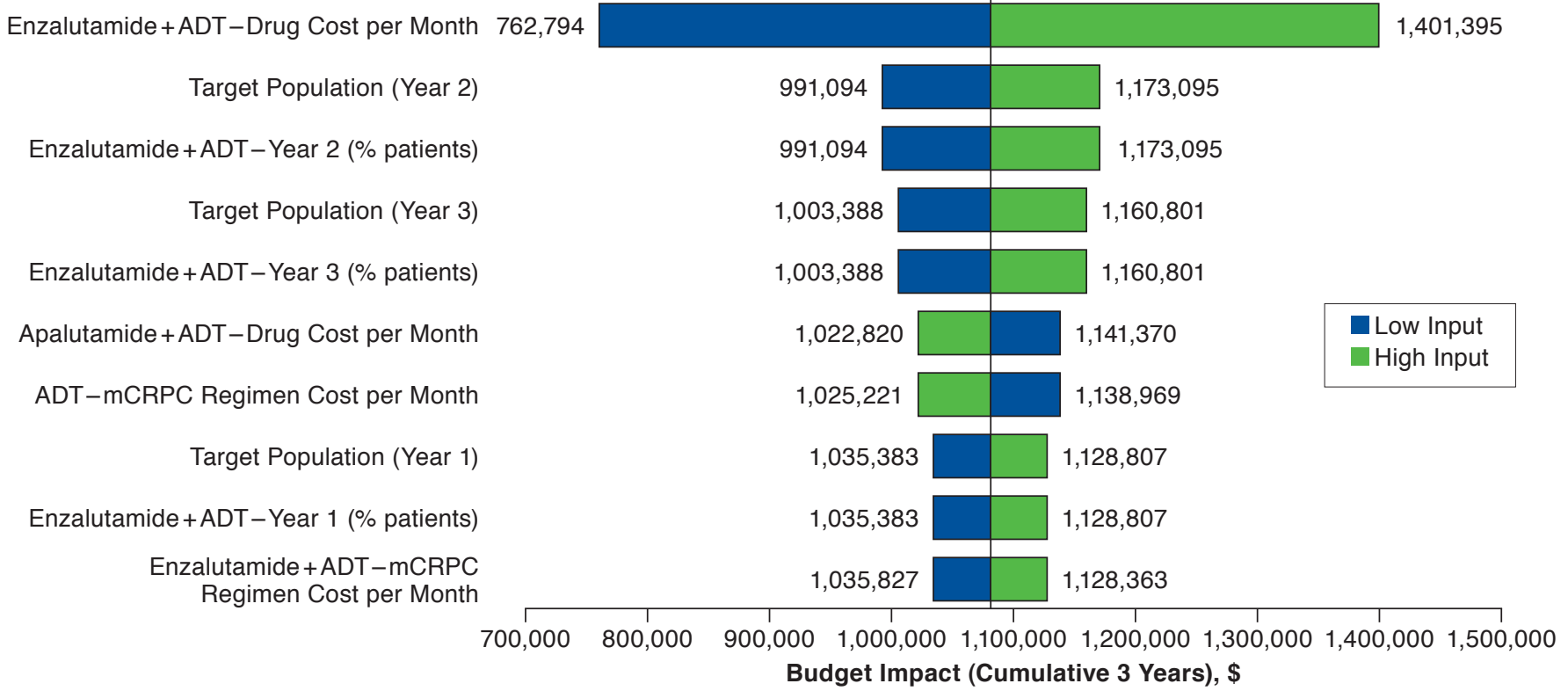

$A D T=$ androgen deprivation therapy; $m C R P C=$ metastatic castration-resistant prostate cancer .

\section{Limitations}

This model was developed in accordance with currently established health economic guidelines, but there are a few limitations that should be noted. ${ }^{16,18}$ First, the budget impact estimates are for a hypothetical 1-million-member health plan and may not be generalizable to other health plans. The model does not include indirect costs such as lost productivity, which may potentially offset the costs of treatments. Results for an actual health plan may differ based on a number of factors, including the size of the treated patient population, costs of treatments and other health care resources, use of patient access restrictions (e.g., cost sharing, prior authorization), and market shares of treatments. To the extent that these factors are known by a health plan, it would be possible to configure the model to obtain a budget impact estimate for a specific health plan.

Second, for this analysis we used the primary clinical trial endpoint from PROSPER-MFS - to define the duration of nmCRPC treatment and the transition to treatment for mCRPC. Patient treatment patterns were modeled using an exponential function with a median MFS of 36.6 months. It was assumed that patients receive nmCRPC treatment until they developed metastatic disease, at which point they immediately initiate $\mathrm{mCRPC}$ treatment. To the extent that some patients may discontinue nmCRPC treatment before developing metastatic disease (e.g., because of adverse events) or that some patients who develop metastatic disease may not immediately initiate
mCRPC treatment, the model may overestimate the total treatment costs of CRPC. Accounting for a treatment-free interval may help to account for this but would have complicated the analysis. The overall effect is that this analysis tends toward overestimating the budget impact.

Third, the model only includes grade 3 and 4 adverse events with an incidence greater than $1 \%$ among the nmCRPC treatments. These adverse events were included because of the potential higher costs of grade 3 and 4 events, which were assumed to involve inpatient hospitalization. More common but lower cost grade 1 and 2 events were not included, nor were adverse events for mCRPC treatments. Monitoring costs, based on product warnings and precautions, were included for nmCRPC treatments but not for mCRPC treatments. In the 1-way sensitivity analysis, none of the adverse events or monitoring costs had an appreciable effect on the results.

Finally, future market share estimates were based on forecasts that are challenging to accurately estimate. To the extent that product uptake is lower or higher, the budget impact could be lower or higher than forecast. The 1-way sensitivity analysis suggests that these parameters are among the more potentially impactful in the model.

\section{Conclusions}

Budget impact models may aid health care decision makers in evaluating new therapies for PC. This model estimated the bud- 
get impact on a U.S. health plan of enzalutamide in combination with ADT for the treatment of patients with high-risk nmCRPC. Over 3 years, with enzalutamide use increasing to $\sim 26 \%$, the budget impact on a PMPM basis was estimated as $\$ 0.028$. Enzalutamide +ADT offers nmCRPC patients a new treatment option with demonstrated clinical benefits, including delayed progression to mCRPC, with a modest budget impact.

\section{Authors}

NEIL M. SCHULTZ, PharmD, MS, Astellas Pharma, Northbrook, Illinois. KEN O'DAY, PhD, MPA, and REBECCA SUGARMAN, BSc, MSc, Xcenda, Palm Harbor, Florida. KRISHNAN RAMASWAMY, PhD, Pfizer, New York.

AUTHOR CORRESPONDENCE: Neil M. Schultz, PharmD, MS, Astellas Pharma, 1 Astellas Way, Northbrook, IL 60062.

Tel.: 224.205.5415; E-mail: neil.schultz@astellas.com.

\section{DISCLOSURES}

This research was sponsored by Astellas Pharma and Pfizer, the codevelopers of enzalutamide. All authors contributed to the development of the manuscript and maintained control over the final content. Schultz is employed by Astellas Pharma and owns stock in Gilead Sciences and Shire. O'Day and Sugarman are employees of Xcenda, which received consultancy fees from Astellas Pharma. Ramaswamy is employed by Pfizer.

A synopsis of the current study was presented in poster format at the AMCP Managed Care \& Specialty Pharmacy Annual Meeting 2019, in San Diego, CA, on March 25-28, 2019.

\section{ACKNOWLEDGMENTS}

Medical writing assistance was provided by Derek Louie, with Xcenda, and editorial assistance was provided by Patrick Gonyo, PhD; Lianne Young, BSc (Hons); Jane Beck; and Lauren Smith, with Complete HealthVizion, all funded by the study sponsors.

\section{DATA-SHARING STATEMENT}

Upon request, and subject to certain criteria, conditions and exceptions (see https://www.pfizer.com/science/clinical-trials/trial-data-and-results for more information), Pfizer will provide access to individual deidentified participant data from Pfizer-sponsored global interventional clinical studies conducted for medicines, vaccines, and medical devices (1) for indications that have been approved in the US and/or EU or (2) in programs that have been terminated (i.e., development for all indications has been discontinued). Pfizer will also consider requests for the protocol, data dictionary, and statistical analysis plan. Data may be requested from Pfizer trials 24 months after study completion. The deidentified participant data will be made available to researchers whose proposals meet the research criteria and other conditions, and for which an exception does not apply, via a secure portal. To gain access, data requestors must enter into a data-access agreement with Pfizer.

\section{REFERENCES}

1. Siegel RL, Miller KD, Jemal A. Cancer statistics, 2019. CA Cancer J Clin. 2019;69(1):7-34.
2. American Cancer Society. Key statistics for prostate cancer. January 8 , 2020. Available at: https://www.cancer.org/cancer/prostate-cancer/about/ key-statistics.html. Accessed January 15, 2020.

3. Mateo J, Fizazi K, Gillessen S, et al. Managing nonmetastatic castrationresistant prostate cancer. Eur Urol. 2019;75(2):285-93.

4. Kirby M, Hirst C, Crawford ED. Characterising the castration-resistant prostate cancer population: a systematic review. Int J Clin Pract. 2011;65(11):1180-92.

5. Xie W, Regan MM, Buyse M, et al. Metastasis-free survival is a strong surrogate of overall survival in localized prostate cancer. J Clin Oncol. 2017;35(27):3097-104.

6. Dieleman JL, Baral R, Birger M, et al. US spending on personal health care and public health, 1996-2013. JAMA. 2016;316(24):2627-46.

7. Stokes ME, Ishak J, Proskorovsky I, Black LK, Huang Y. Lifetime economic burden of prostate cancer. BMC Health Serv Res. 2011;11:349.

8. Valderrama A, Tangirala K, Babajanyan S, et al. Treatment, healthcare resource utilization, and costs associated with non-metastatic and metastatic castration-resistant prostate cancer: a claims analysis. J Clin Oncol. 2017;35(Suppl 15):e18341.

9. American Urological Association. Castration-resistant prostate cancer: AUA guideline 2018. 2018. Available at: https://www.auanet.org/Documents/ Guidelines/PDF/GUI-18-3248\%20CRPC\%20Guideline\%20Algorithm\%20 UpdateF(0).pdf. Accessed January 15, 2020.

10. Xtandi (enzalutamide) capsules, for oral use. Astellas Pharma US, Inc. July 2018. Available at: https://www.accessdata.fda.gov/drugsatfda_docs/ label/2018/203415s014lbl.pdf. Accessed January 15, 2020.

11. Beer TM, Armstrong AJ, Rathkopf DE, et al. Enzalutamide in metastatic prostate cancer before chemotherapy. N Engl J Med. 2014;371(5):424-33.

12. Scher HI, Fizazi K, Saad F, et al. Increased survival with enzalutamide in prostate cancer after chemotherapy. N Engl J Med. 2012;367(13):1187-97.

13. Hussain M, Fizazi K, Saad F, et al. Enzalutamide in men with nonmetastatic, castration-resistant prostate cancer. N Engl J Med. 2018;378(26):2465-74.

14. Smith MR, Saad F, Chowdhury S, et al. Apalutamide treatment and metastasis-free survival in prostate cancer. N Engl J Med. 2018;378(15):1408-18.

15. Penson DF, Armstrong AJ, Concepcion R, et al. Enzalutamide versus bicalutamide in castration-resistant prostate cancer: the STRIVE trial. J Clin Oncol. 2016;34(18):2098-106.

16. Academy of Managed Care Pharmacy. The AMCP Format for Formulary Submissions (version 4.1). December 2019. Available at: https://www.amcp. org/sites/default/files/2019-12/AMCP_Format\%204.1_1219_final.pdf. Accessed January 15, 2020.

17. Mauskopf JA, Sullivan SD, Annemans L, et al. Principles of good practice for budget impact analysis: report of the ISPOR Task Force on good research practices_budget impact analysis. Value Health. 2007;10(5):336-47.

18. Sullivan SD, Mauskopf JA, Augustovski F, et al. Budget impact analysis-principles of good practice: report of the ISPOR 2012 Budget Impact Analysis Good Practice II Task Force. Value Health. 2014;17(1):5-14.

19. Truven Health Analytics, IBM Watson Health. Micromedex RED BOOK Online. Database. February 7, 2017. Available at: http://truvenhealth.com/ Products/Micromedex/Product-Suites/Clinical-Knowledge/RED-BOOK. Accessed January 15, 2020.

20. National Cancer Institute. SEER* Explorer: surveillance, epidemiology, and end results program. April 15, 2019. Available at: https://seer. cancer.gov/explorer/application.php?site $=66 \&$ data_type $=4 \& g r a p h$ type $=2 \&$ compareBy=time_interval $\&$ hdn_sex $=2 \&$ chk_race_l=1\&chk_ age_range_l=1\&chk_stage_106=106\&chk_time_interval_l=1\&chk_time_ interval_5=5\&advopt_precision=1\&advopt_display=1\&advopt_show_ ci=on\&showDataFor=race_1_and_age_range_1_and_stage_106. Accessed January 15, 2020. 
21. Centers for Medicare \& Medicaid Services. Clinical laboratory fee schedule, 2018. January 2018. Available at: https://www.cms.gov/Medicare/ Medicare-Fee-for-Service-Payment/ClinicalLabFeeSched/Clinical-LaboratoryFee-Schedule-Files-Items/18CLAB.html. Accessed January 15, 2020.

22. Centers for Medicare \& Medicaid Services. Physician fee schedule, 2018 October 4, 2019. Available at: https://www.cms.gov/apps/physician-feeschedule/. Accessed January 15, 2020.

23. National Cancer Institute. Common terminology criteria for adverse events (CTCAE; version 5.0). November 27, 2017. Available at: https://ctep. cancer.gov/protocoldevelopment/electronic_applications/ctc.htm. Accessed January 15, 2020.

24. Agency for Healthcare Research and Quality. HCUPnet: Healthcare Cost and Utilization Project. Available at: https://hcupnet.ahrq.gov. Accessed January 15, 2020.

25. Burns ER, Stevens JA, Lee R. The direct costs of fatal and non-fatal falls among older adults-United States. J Safety Res. 2016;58:99-103.

26. U.S. Bureau of Labor Statistics. Consumer Price Index. 2018. Available at: https://data.bls.gov/cgi-bin/surveymost?cu. Accessed January 15, 2020.

27. Wallis CJD, Chandrasekar T, Goldberg H, et al. Advanced androgen blockage in nonmetastatic castration-resistant prostate cancer: an indirect comparison of apalutamide and enzalutamide. Eur Urol Oncol. 2018;1(3):238-41

28. Luo J, Graff JN. Impact of enzalutamide on patient-related outcomes in metastatic castration-resistant prostate cancer: current perspectives. Res Urol. 2016;8:217-24.

29. Almeida DVP, de Oliveira CZ, Mariano RC, et al. Non-metastatic castration-resistant prostate cancer (nmCRPC): meta-analysis of efficacy and safety with novel hormonal agents apalutamide and enzalutamide. Ann Oncol. 2018;29(Suppl 8):viii271-302.

30. Grochtdreis T, König HH, Dobruschkin A, von Amsberg G, Dams J. Cost-effectiveness analyses and cost analyses in castration-resistant prostate cancer: a systematic review. PLoS One. 2018;13(12):e0208063.

31. Massoudi M, Balk M, Yang H, et al. Number needed to treat and associated incremental costs of treatment with enzalutamide versus abiraterone acetate plus prednisone in chemotherapy-naive patients with metastatic castration-resistant prostate cancer. J Med Econ. 2017;20(2):121-28.

32. Bui CN, O’Day K, Flanders S, et al. Budget impact of enzalutamide for chemotherapy-naive metastatic castration-resistant prostate cancer. J Manag Care Spec Pharm. 2016;22(2):163-70. Available at: https://www.jmcp.org/ doi/10.18553/jmcp.2016.22.2.163.
33. Institute for Clinical and Economic Review. Antiandrogens for nonmetastatic castration-resistant prostate cancer: effectiveness and value. Final evidence report. October 4, 2018. Available at: https://icer-review. org/wp-content/uploads/2018/02/ICER_Prostate_Cancer_Final_Evidence_ Report_100418.pdf. Accessed January 15, 2020.

34. Scher HI, Solo K, Valant J, Todd MB, Mehra M. Prevalence of prostate cancer clinical states and mortality in the United States: estimates using a dynamic progression model. PLoS One. 2015;10(10):e0139440.

35. Liede A, Arellano J, Hechmati G, Bennet B, Wong S. International prevalence of nonmetastatic (M0) castration-resistant prostate cancer (CRPC). J Clin Oncol. 31(Suppl 15):e16052.

36. U.S. Census Bureau. Annual estimates of the resident population by single year of age and sex for the United States: April 1, 2010 to July 1, 2018 (NC-EST2018-AGESEX-RES). 2016. Available at: https://www.census.gov/ data/tables/time-series/demo/popest/2010s-national-detail.html. Accessed January 30, 2020

37. Noone AM, Howlader N, Krapch M, et al. SEER Cancer statistics review, 1975-2015. September 10, 2018. Available at: https://seer.cancer.gov/ csr/1975_2015/results_merged/sect_04_breast.pdf. Accessed January 15, 2020.

38. Nakabayashi M, Hayes J, Taplin ME, et al. Clinical predictors of survival in men with castration-resistant prostate cancer: evidence that Gleason score 6 cancer can evolve to lethal disease. Cancer. 2013;119(16):2990-98.

39. Hernandez RK, Cetin K, Pirolli M, et al. Estimating high-risk castration resistant prostate cancer (CRPC) using electronic health records. Can J Urol. 2015;22(4):7858-64.

40. Sivanandam A, Siva S, Bhandari M, Menon M. Variance inflation in sequential calculations of body surface area, plasma volume, and prostatespecific antigen mass. BJU Int. 2008;102(11):1573-80.

41. Erleada (apalutamide) tablets, for oral use. Janssen Products. February 2018. Available at: https://www.accessdata.fda.gov/drugsatfda_docs/ label/2018/210951s000lbl.pdf. Accessed January 15, 2020.

42. Casodex (bicalutamide) tablet, for oral use. AstraZeneca Pharmaceuticals. October 2017. Available at: https://www.accessdata.fda.gov/drugsatfda_docs/ label/2017/020498s028lbl.pdf. Accessed January 15, 2020.

43. Lupron Depot (leuprolide acetate for depot suspension). AbbVie. June 2014. Available at: https://www.accessdata.fda.gov/drugsatfda_docs/label/20 14/020517s036_019732s041lbl.pdf. Accessed January 15, 2020. 


\section{APPENDIX A Key Model Assumptions}

- All patients receive gonadotropin-releasing hormone therapy, and no patients underwent prior bilateral orchiectomy.

- Treatment costs for nonmetastatic patients were assumed to apply during the period of MFS based on clinical trial data. For bicalutamide + ADT, it was assumed that the median MFS was identical to ADT based on a similar hazard ratio for both treatments compared with enzalutamide in the PROSPER and STRIVE trials.

- All patients were assumed to receive the same ADT treatment.

- For patients who progress to metastatic disease, no survival differences were assumed among metastatic treatments.

- Adverse event costs were based on the assumption that grade 3 and 4 events would require hospitalization, consistent with the National Cancer Institute Common Terminology Criteria for Adverse Events definitions.

- Adverse event rates apply to patients over the course of treatment, as defined by the median MFS.

- Enzalutamide's market share is obtained from the other comparators in proportion to their market share in a scenario without enzalutamide.

$A D T=$ androgen deprivation; $M F S=$ metastasis-free survival . 


\section{APPENDIX B Adverse Events and Monitoring (Applied to nmCRPC Only) ${ }^{24,25}$}

\begin{tabular}{|c|c|c|c|c|c|}
\hline Adverse Event & Cost/Event, ${ }^{\text {a } \$}$ & Enzalutamide + ADT, \% & Apalutamide + ADT, \% & Bicalutamide + ADT, \% & ADT, \% \\
\hline Anemia & 7,157 & NR & NR & 5.1 & NR \\
\hline Asthenia & 7,383 & 1.2 & NR & NR & 0.2 \\
\hline Decreased appetite & 9,847 & 0.2 & NR & 2.0 & 0.2 \\
\hline Decreased weight & 8,838 & 0.2 & 1.1 & NR & 0.0 \\
\hline Diarrhea & 7,769 & 0.3 & 1.0 & 1.0 & 0.4 \\
\hline Fall & 11,115 & 1.3 & 1.7 & 1.5 & 0.6 \\
\hline Fatigue & 7,383 & 2.9 & 0.9 & 2.5 & 0.6 \\
\hline Fracture & 16,404 & 2.0 & 2.7 & NR & NR \\
\hline Hematuria & 6,765 & 1.7 & NR & NR & 2.8 \\
\hline Hypertension & 6,606 & 4.6 & 14.3 & 1.5 & 2.2 \\
\hline Major adverse cardiovascular event & 15,987 & 3.7 & NR & NR & 1.7 \\
\hline Rash & 5,418 & NR & 5.2 & NR & NR \\
\hline Seizure & 7,589 & 0.4 & 0.2 & NR & NR \\
\hline Urinary retention & 6,877 & 0.4 & NR & NR & 1.1 \\
\hline Urinary tract infection & 7,420 & 0.8 & NR & 3.0 & 0.6 \\
\hline
\end{tabular}

aValues were inflated to 2018 U.S. dollars.

$A D T=$ androgen deprivation therapy; $n m C R P C=$ nonmetastatic castration-resistant prostate cancer; $N R=$ not reported. 
Budget Impact of Enzalutamide for Nonmetastatic Castration-Resistant Prostate Cancer

\section{APPENDIX C Market Share}

\begin{tabular}{|c|c|c|c|c|}
\hline Market Share & Enzalutamide + ADT, \% & Apalutamide + ADT, \% & Bicalutamide + ADT, \% & ADT, $\%$ \\
\hline \multicolumn{5}{|c|}{ Without enzalutamide } \\
\hline Year 1 & 0.0 & 5.7 & 28.5 & 65.8 \\
\hline Year 2 & 0.0 & 16.0 & 25.4 & 58.6 \\
\hline Year 3 & 0.0 & 27.0 & 22.1 & 50.9 \\
\hline \multicolumn{5}{|c|}{ With enzalutamide } \\
\hline Year 1 & 5.7 & 5.4 & 26.9 & 62.0 \\
\hline Year 2 & 15.9 & 13.5 & 21.4 & 49.3 \\
\hline Year 3 & 25.8 & 20.0 & 16.4 & 37.8 \\
\hline
\end{tabular}

